\title{
ON SELF-MAPS WHICH INDUCE IDENTITY AUTOMORPHISMS OF HOMOLOGY GROUPS
}

\author{
PETAR PAVEŠIĆ* \\ Department of Mathematics, University of Ljubljana, Jadranska 19, Ljubljana, Slovenia \\ e-mail:petar.pavesic@fmf.uni-lj.si
}

(Received 5 July, 1999)

\begin{abstract}
We derive a finite normal series for the group $\operatorname{Aut}_{*}(X)$ of selfhomotopy equivalences which induce identity automorphisms of homology groups, where $X$ is a countable, simply-connected and finite-dimensional CW-complex.
\end{abstract}

1991 Mathematics Subject Classification. Primary: 55P10.

1. Introduction. Let $X$ be a pointed CW-complex and let $\operatorname{Aut}(X)$ denote the set of homotopy classes of self-maps of $X$ that are homotopy equivalences. This set is a group, the group of self-homotopy equivalences, with respect to the operation induced by the composition of maps. The survey paper [1] and the book [8] give an idea of the extensive literature on these groups. Still, their structure is very often unknown, and one of the main difficulties originates from the fact that a cellular (or even a homology) decomposition of $X$ does not lead to a corresponding decomposition of $\operatorname{Aut}(X)$. In fact, there are elementary examples when a self-equivalence of a space cannot be represented by a cellular map whose restrictions on skeletons are also selfequivalences (cf. Remark 1.1 of [7]).

The main purpose of this article is to show that the situation is more favorable when dealing with $\operatorname{Aut}_{*}(X)$, the subgroup of $\operatorname{Aut}(X)$ consisting of classes that induce identity automorphisms of homology groups (or, in other words, with the kernel of the representation $\operatorname{Aut}(X) \longrightarrow$ Aut $H_{*}(X)$ on homology). The crucial step is Theorem 1 that for a large class of spaces $X$ any element of $\operatorname{Aut}_{*}(X)$ can be represented by a cellular map which yields a self-equivalence inducing identity on homology when restricted to any skeleton of $X$. With this fact at hand it is easy to derive a finite normal series for $\operatorname{Aut}_{*}(X)$. The existence of such a normal series paves the way for a construction of a spectral sequence converging to $\operatorname{Aut}_{*}(X)$ that we are going to consider in a forthcoming paper.

We wish to remark that much of this work was motivated by the proof of the main theorem in [5] and that we used similar arguments in proving our results.

2. A normal series for $\operatorname{Aut}_{*}(X)$. Let $\varphi: V \rightarrow A$ be a map from a wedge of $n$ dimensional $(n \geq 2)$ spheres $V$ to an $n$-dimensional, 1-connected $\mathrm{CW}$-complex $A$, and let $C_{\varphi}$ be the mapping cone of $\varphi$. By Theorem 7.3' of [4], for every self-map $f$ of $C_{\varphi}$ there are self-maps $f_{A}$ and $f_{V}$ of $A$ and $V$ respectively, such that the following diagram commutes up to homotopy.

* Partly supported by the Ministry for Science and Technology of the Republic of Slovenia, Grant No. J1-0885-0101-98. 


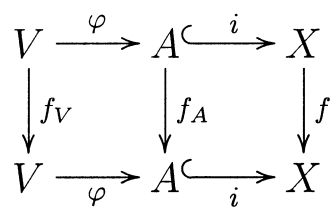

Note that maps $f_{A}$ in $f_{V}$ are by no means unique. For an $f \in \operatorname{Aut}_{*}\left(C_{\varphi}\right)$ a chosen $f_{A}$ will not in general be an element of $\operatorname{Aut}_{*}(A)$. However, under suitable assumptions the choice can be modified in order to obtain maps $f_{A}^{\prime}$ and $f_{V}^{\prime}$ that fit the above diagram, and such that $f_{A}^{\prime} \in \operatorname{Aut}_{*}(A)$. This can be achieved by means of an action of $[A, V]$ on $[A, A]$ that we describe in the following paragraph.

Let $F$ be the homotopy fibre of the projection $C_{\varphi} \rightarrow C_{\varphi} / A \simeq \Sigma V$. The homotopy fibre of $F \hookrightarrow C_{\varphi}$ is homotopy equivalent to $\Omega \Sigma V$. By the universal property of Puppe fibration sequences there are maps $j, k$ such that the diagram

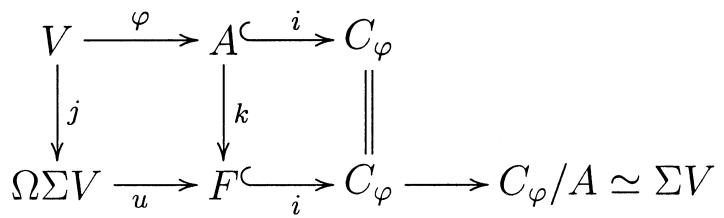

commutes up to homotopy. Note that $j: V \rightarrow \Omega \Sigma V$ is the usual homology suspension map.

Lemma 1. The maps $j$ and $k$ induce bijections

$$
j^{*}=j \circ-:[A, V] \rightarrow[A, \Omega \Sigma V]
$$

and

$$
k^{*}=k^{\circ}-:[A, A] \rightarrow[A, F] .
$$

Proof. By Lemma 3.1 of [3] the maps $j$ and $k$ are $n+1$-connected, and hence induce the equivalence of the respective $n$-types. Since $\operatorname{dim}(A) \leq n$ we get that $j^{*}$ and $k^{*}$ are bijections.

The lifting of paths induces an action of $\Omega \Sigma V$ on $F$ that we denote by $\mu: \Omega \Sigma V \times F \rightarrow F$. Through the above bijections this yields an action of $[A, V]$ on $[A, A]$ : given $\alpha \in[A, V]$ and $g \in[A, A]$ define $\alpha \cdot g$ to be $\left(k^{*}\right)^{-1}$ of the composition

$$
A \stackrel{(\alpha, g)}{\longrightarrow} V \times A \stackrel{j \times k}{\longrightarrow} \Omega \Sigma V \times F \stackrel{\mu}{\longrightarrow} F .
$$

Note that $i \circ(\alpha \cdot g) \simeq i \cdot g: A \rightarrow C_{\varphi}$ for every $g: A \rightarrow A$.

Lemma 2. The action of $\alpha \cdot g$ on the homology of $A$ is given by $(\alpha \cdot g)_{*}=\varphi_{*} \alpha_{*}+g_{*}$. In other words, $(\alpha \cdot g)_{* q}=g_{* q}$ when $q \neq n$, and $(\alpha \cdot g)_{* n}=\varphi_{* n} \alpha_{* n}+g_{* n}$.

Proof. Since $A$ is $n$-dimensional, it is sufficient to consider the case $q \leq n$. By the considerations above $H_{q}(\Omega \Sigma V)=0$ for $q<n$ and, by the Eilenberg-Zilber theorem, we get that for $q \leq n$ there is a direct sum representation $H_{q}(\Omega \Sigma V \times F)=$ 
$H_{q}(\Omega \Sigma V) \oplus H_{q}(F)$ induced by the inclusions of $\Omega \Sigma V$ and $F$ in the product. Hence we have the following commutative diagram $(q \leq n)$.

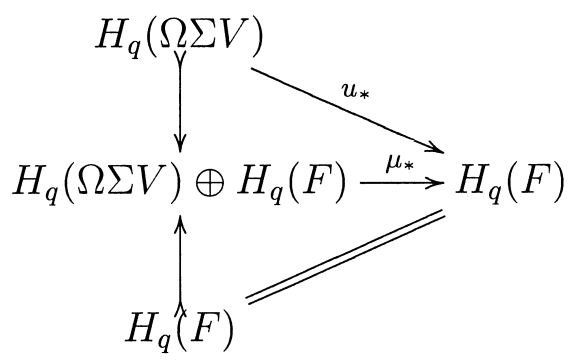

We conclude that in dimensions under consideration the effect of $\mu_{*}$ on an element $(x, y) \in H_{q}(\Omega \Sigma V \times F)$ is given by $\mu_{*}(x, y)=u_{*}(x)+y$. The action of

$$
\alpha \cdot g=\left(k^{*}\right)^{-1}[\mu \circ(j \times k) \circ(\alpha, g)]
$$

on $x \in H_{q}(A)$ is now easily computed:

$$
\mu_{*} \circ(j \times k)_{*} \circ(\alpha, g)_{*}(x)=u_{*} j_{*} \alpha_{*}(x)+k_{*} g_{*}(x)=k_{*} \varphi_{*} \alpha_{*}(x)+k_{*} g_{*}(x) .
$$

As $k_{*}$ is an isomorphism when $q \leq n$, the assertion of the lemma is proved.

In order to prove our main result we need another piece of structure. Let us say that the attaching map $\varphi: V \rightarrow A$ is canonical if the wedge of spheres $V$ can be decomposed into two sub-wedges $V=V_{r} \vee V_{g}$ such that $\left(\left.\varphi\right|_{V_{r}}\right)_{*}: H_{n}\left(V_{r}\right) \rightarrow H_{n}(A)$ is injective and $\varphi\left(V_{g}\right)$ is contained in the $(n-1)$-skeleton of $A$ (and hence $\left.\left(\left.\varphi\right|_{V_{g}}\right)_{*}=0\right)$. When this is the case the exact sequence

$$
0 \longrightarrow H_{n+1}\left(C_{\varphi}\right) \longrightarrow H_{n}(V) \stackrel{\varphi_{*}}{\longrightarrow} H_{n}(A) \longrightarrow H_{n}\left(C_{\varphi}\right) \longrightarrow 0
$$

which determines the homology of $C_{\varphi}$ in dimensions $n$ and $n+1$, can be decomposed into two shorter exact sequences

$$
0 \longrightarrow H_{n+1}\left(C_{\varphi}\right) \stackrel{\cong}{\longrightarrow} H_{n}\left(V_{g}\right) \longrightarrow 0
$$

and

$$
0 \longrightarrow H_{n}\left(V_{r}\right) \stackrel{\left(\left.\varphi\right|_{V_{r}}\right)_{*}}{\longrightarrow} H_{n}(A) \longrightarrow H_{n}\left(C_{\varphi}\right) \longrightarrow 0
$$

A CW-complex has a canonical decomposition if the attaching maps in all dimensions are canonical. By Theorem 2.3 of [7] every simply connected countable CWcomplex $X$ has the cellular homotopy type of a CW-complex with a canonical decomposition $Y$; i.e. there are cellular maps $f: X \rightarrow Y$ and $g: Y \rightarrow X$, such that $f \circ g$ and $g \circ f$ are homotopic to $1_{Y}$ and $1_{X}$ by cellular homotopies.

Lemma 3. Assume that the attaching map $\varphi: V \rightarrow A$ is canonical. Then, for every map $f: C_{\varphi} \rightarrow C_{\varphi}$ satisfying $f_{* n}=1_{H_{n}\left(C_{\varphi}\right)}$, there exists a map $\bar{f}: A \rightarrow A$ such that if $\simeq f i$, $\bar{f}_{* n}=1_{H_{n}(A)}$ and $\bar{f}_{* q}=f_{* q}$ for $q<n$. 
Proof. As we mentioned at the begining of this section, there are maps $f_{A}$ and $f_{V}$ with $i \circ f_{A}=f \circ i$ and $\varphi \circ f_{V} \simeq f_{A} \circ \varphi$. Since $\varphi$ is canonical, we obtain the following commutative diagram with exact rows (where $l: V_{r} \hookrightarrow V$ and $\pi: V \rightarrow V_{r}$ are the natural inclusion and projection respectively).

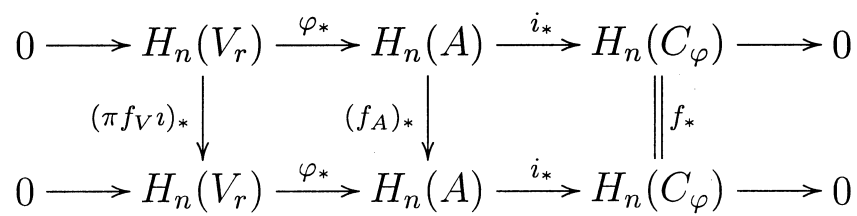

The equality

$$
i_{*}\left(1-\left(f_{A}\right)_{*}\right)=i_{*}-i_{*}\left(f_{A}\right)_{*}=0
$$

implies that $s:=\varphi_{*}^{-1} \circ\left(1-\left(f_{A}\right)_{*}\right)$ is a well-defined homomorphism from $H_{n}(A)$ to $H_{n}\left(V_{r}\right)$. Using Hopf's theorem and the fact that $H_{n}(A)$ is a free group we deduce the existence of a map $\alpha: A \rightarrow V$ such that $\alpha_{* n}=s$. Finally, $\bar{f} \alpha \cdot f_{A}$ is a map satisfying the conditions of the lemma.

Using this lemma as inductive step we can prove the following result.

Theorem 1. Assume that $X$ is countable, simply connected and finite-dimensional. Then every element of $\mathrm{Aut}_{*}(X)$ can be represented by a cellular map whose restriction to every skeleton $X^{(q)}$ belongs to $\operatorname{Aut}_{*}\left(X^{(q)}\right)$.

Proof. Let $h: X \rightarrow Y$ be a cellular homotopy equivalence, where $Y$ is an $n$ dimensional CW-complex with a canonical decomposition, and let $\tilde{h}$ be a cellular homotopy inverse of $h$. For every map $f: X \rightarrow X$ representing a class in $\operatorname{Aut}_{*}(X)$ the composition $g:=h \circ f \circ \tilde{h}$ represents a class in $\operatorname{Aut}_{*}(Y)$.

We shall use induction to prove that there is a cellular map $\bar{g}$ homotopic to $g$, such that $\left.\bar{g}\right|_{Y^{(q)}} \in \operatorname{Aut}_{*}\left(Y^{(q)}\right)$, for every $q$. Assume inductively that there is a cellular map $g: Y \rightarrow Y$, such that $\left.g\right|_{Y^{(n-q)}} \in \operatorname{Aut}_{*}\left(Y^{(n-q)}\right)$, for $q=0,1, \ldots, k-1$. As the inclusion $i_{n-k}: Y^{(n-k)} \hookrightarrow Y^{(n-k+1)}$ is a cofibration, the map $\bar{g}_{n-k} \in \operatorname{Aut}_{*}\left(Y^{(n-k)}\right)$ satisfying $i_{n-k} \circ \bar{g}_{n-k} \simeq g \circ i_{n-k}$, which exists by the previous lemma, can be extended to a $\operatorname{map} \bar{g}_{n-k+1} \in \operatorname{Aut}_{*}\left(Y^{(n-k+1)}\right)$ homotopic to $\left.g\right|_{Y^{(n-k+1)}}$. Iterations of that construction eventually yield a cellular map $\bar{g} \in \operatorname{Aut}_{*}(Y)$ with the property $\left.\bar{g}\right|_{Y^{(n-q)}} \in \operatorname{Aut}_{*}\left(Y^{(n-q)}\right)$, for $q=0,1, \ldots, k$. Since the assertion for $g$ is obviously true when $k=0$, the claim is proved.

The map $\bar{f}:=\tilde{h} \circ \bar{g}^{\circ} h$ is homotopic to $f$, and due to the cellularity of $h$ and $\tilde{h}$, $\left.f\right|_{X^{(q)}} \in \operatorname{Aut}_{*}\left(X^{(q)}\right)$.

Let us denote by $G_{q}=G_{q}(X)$ the subset of $\operatorname{Aut}_{*}(X)$ whose elements are represented by maps $f$ with the property $\left.f\right|_{X^{(q)}}=1_{X^{(q)}}$. We shall show that $G_{q}$ is a subgroup of $\operatorname{Aut}_{*}(X)$.

Lemma 4. Let $\varphi: V \rightarrow A$ be as above. If $f: C_{\varphi} \rightarrow C_{\varphi}$ and $\left.f\right|_{A}: A \rightarrow A$ are homotopy equivalences, then $f$ has a homotopy inverse $g$ such that $\left.g\right|_{A}$ is a homotopy inverse of $\left.f\right|_{A}$. 
Proof. Consider the following diagram:

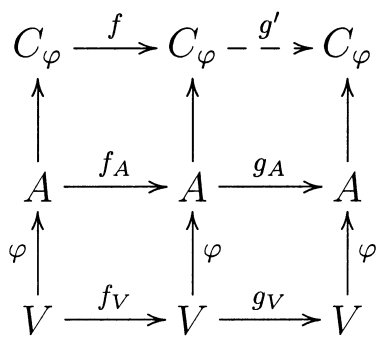

where $g_{V}$ and $g_{A}$ are homotopy inverses of $f_{V}$ and $f_{A}$ respectively. Since the lower right square is homotopy commutative it induces a homotopy equivalence $g^{\prime}: C_{\varphi} \rightarrow C_{\varphi}$, such that $\left.\left(g^{\prime} \circ f\right)\right|_{A} \simeq 1_{A}$. By Lemma 1.7 and Lemma 1.8 of [6], the set of self-homotopy equivalences of $C_{\varphi}$ that restrict to the identity on $A$ is a subgroup of $\operatorname{Aut}(X)$, so there is a homotopy inverse $h$ for $g^{\prime} \circ f$, such that $\left.h\right|_{A} \simeq 1_{A}$. Then the map $g:=h \circ g^{\prime}$ satisfies the requirements of the lemma.

Using this lemma as inductive step we can construct, for every element of $G_{q}$, an inverse, which is also in $G_{q}$.

Corollary 1. $G_{q}$ is a subgroup of $\operatorname{Aut}_{*}(X)$ for all $q$.

The same inductive procedure yields the following result also.

Corollary 2. Let $X$ be a simply connected $C W$-complex, and let $f: X \rightarrow X$ be such that $\left.f\right|_{X^{(q)}} \in \operatorname{Aut}_{*}\left(X^{(q)}\right)$, for all $q$. Then there exists a map $g: X \rightarrow X$, such that $\left.g\right|_{X^{(q)}}$ is a homotopy inverse of $\left.f\right|_{X^{(q)}}$.

The proof of our main result is now at hand.

Theorem 2. Let $X$ be a countable, simply connected $n$-dimensional $C W$-complex. Then $G_{q}$ is a normal subgroup of $\operatorname{Aut}_{*}(X)$, for every $q$. Moreover,

$$
1 \triangleleft G_{n-1} \triangleleft \ldots \triangleleft G_{2} \triangleleft \mathrm{Aut}_{*} X
$$

is a finite normal series for $\operatorname{Aut}_{*}(X)$.

Proof. Let $[f] \in G_{q}$ and $[g] \in \operatorname{Aut}_{*}(X)$ : we must prove that $[g][f][g]^{-1} \in G_{q}$.

Because of Theorem 1 and Corollary 2 we can assume that $\left.g\right|_{X^{(q)}} \in \operatorname{Aut}_{*}\left(X^{(q)}\right)$, and that $[g]^{-1}$ is represented by a map $\tilde{g}$, such that $\left.\tilde{g}\right|_{X^{(q)}}$ is a homotopy inverse of $\left.g\right|_{X^{(q)}}$.

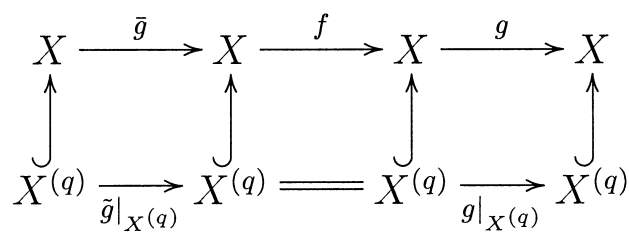


implies that $\left.(g \circ f \circ \tilde{g})\right|_{X^{(q)}}$ is homotopic to $1_{X^{(q)}}$. As the inclusion of $X^{(q)}$ in $X$ is a cofibration, $g \circ f \circ \tilde{g}$ is homotopic to a map that restricts to $1_{X^{(q)}}$; hence it belongs to a class in $G_{q}$, which proves the normality.

3. Applications. As already mentioned, the existence of a normal series is a necessary condition if one wants to construct a spectral sequence for a noncommutative group. In the Shih's spectral sequence (see [9]) such a normal series for $\operatorname{Aut}(X)$ is obtained for free, due to the naturality of the Postnikov's decomposition. The apparent impossibility to construct a normal series for $\operatorname{Aut}(X)$ corresponding to a cellular decomposition is a real obstacle for the study of this group by means of spectral sequences.

However, spectral sequences for $\operatorname{Aut}_{*}(X)$ will be treated elsewhere, but there are also some immediate implications, which we consider in this section. In the presence of a normal series it is natural to ask what are its subquotients. The description of these turns out to be quite complicated but nonetheless, some estimates are possible.

When $X$ is a subspace of $Y$ let us denote by $\operatorname{aut}_{* X}(Y)$ the space consisting of selfmaps of $Y$ that induce identity automorphisms of homology groups and which restrict to the identity on $X$, and let $\operatorname{Aut}_{* X}(Y):=\pi_{0}\left(\operatorname{aut}_{* X}(Y)\right)$.

Lemma 5. Let $X$ be as in Theorem 2. For a fixed $q$, if the group $\operatorname{Aut}_{* X^{(q)}}\left(X^{(q+1)}\right)$ is trivial, then so is the quotient $G_{q} / G_{q+1}$ (i.e. $G_{q+1}=G_{q}$ ).

Proof. Every element of $G_{q}$ can be represented by a cellular map $f$ such that $\left.f\right|_{X^{(p)}} \in \operatorname{Aut}_{*}\left(X^{(p)}\right)$, for every $p$, and $\left.f\right|_{X^{(q)}}=1_{X^{(q)}}$. The restriction $\left.f\right|_{X^{(q+1)}}$ represents an element of $\operatorname{Aut}_{* X^{(q)}}\left(X^{(q+1)}\right)$, which by the assumptions is trivial, and so there is a homotopy that is fixed on $X^{(q)}$ between $\left.f\right|_{X^{(q+1)}}$ and the identity. As $X^{(q+1)} \hookrightarrow X$ is a cofibration, this homotopy can be extended over all of $X$; therefore $f$ represents an element in $G_{q+1}$.

Consider a canonical decomposition of a CW-complex $X$ : the skeleton $X^{(q+1)}$ is the mapping cone of the attaching map $\varphi_{q}: V^{q} \rightarrow X^{(q)}$, where $V^{q}=V_{g}^{q} \vee V_{g}^{q}$ is a wedge of $q$-dimensional spheres. The application of the functor $\left[-, X^{(q+1)}\right]$ to the Puppe cofibration sequence

$$
V^{q} \stackrel{\varphi_{q}}{\longrightarrow} X^{(q)} \stackrel{i}{\longrightarrow} X^{(q+1)} \stackrel{p}{\longrightarrow} \Sigma V^{q}
$$

yields an exact sequence of pointed sets

$$
\left[\Sigma V^{q}, X^{(q+1)}\right] \stackrel{p^{*}}{\longrightarrow}\left[X^{(q+1)}, X^{(q+1)}\right] \stackrel{i^{*}}{\longrightarrow}\left[X^{(q)}, X^{(q+1)}\right] .
$$

Hence a self-map of $X^{(q+1)}$ restricting to the identity on $X^{(q)}$ corresponds to an element coming from $\left[\Sigma V_{q}, X^{(q+1)}\right]$. In $\S 1$ of [6] it is shown that $p^{*}(f)$ of an $f: \Sigma V_{q} \rightarrow X^{(q+1)}$ is homotopic to the composition

$$
X^{(q+1)} \stackrel{v}{\longrightarrow} X^{(q+1)} \vee \Sigma V^{q} \stackrel{1 \vee f}{\longrightarrow} X^{(q+1)} \vee X^{(q+1)} \stackrel{F}{\longrightarrow} X^{(q+1)},
$$

where $v$ is the standard coaction and $F$ is the folding map. An elementary computation shows that on homology $\left(p^{*}(f)\right)_{*}=1+f_{*} p_{*}$, which implies that the elements of $\mathrm{Aut}_{* X^{(q)}}\left(X^{(q+1)}\right)$ are in bijection with classes in $\left[\Sigma V^{q}, X^{(q+1)}\right]$, such that $f \circ p$ induce 
trivial homomorphisms in homology. Clearly, every map $f: V_{r}^{q} \rightarrow X^{(q+1)}$ satisfies this condition, while for a map $f: V_{g}^{q} \rightarrow X^{(q+1)}$ the condition $f_{*} p_{*}=0$ is equivalent to $f_{*}=0$. The commutativity of the following diagram (in which vertical maps are Hurewicz homomorphisms)

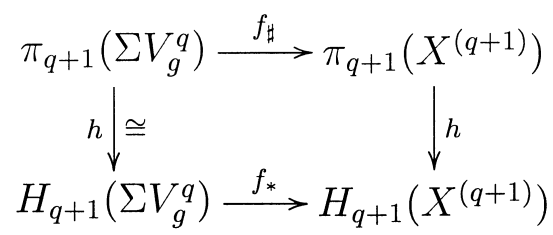

implies that maps with $f_{*}=0$ are in bijection with elements of the kernel of the Hurewicz homomorphism $h: \pi_{q+1}\left(X^{(q+1)}\right) \rightarrow H_{q+1}\left(X^{(q+1)}\right)$.

We can now formulate some conditions for the triviality of $\operatorname{Aut}_{*}(X)$.

Theorem 3. Let $X$ be a countable and simply connected $C W$-complex. The group $\operatorname{Aut}_{*}(X)$ is trivial, provided that the following conditions are satisfied:

(a) the Hurewicz homomorhpism $h: \pi_{q}\left(X^{(q)}\right) \rightarrow H_{q}\left(X^{(q)}\right)$ is injective whenever $H_{q}(X)$ is nontrivial;

(b) $\pi_{q+1}\left(X^{(q+1)}\right)=0$ whenever $H_{q}(X)$ is not free.

Proof. Observe that (up to a cellular homotopy equivalence) there exists a canonical decomposition for $X$, such that $V_{g}^{q}$ is nontrivial only when $H_{q}(X) \neq 0$, and $V_{r}^{q}$ is nontrivial only when $H_{q-1}(X)$ is not free. Indeed, we begin with a homology decomposition for $X$, for which the above holds, and then use the fact that in the construction of the corresponding canonical decomposition no new cells are introduced (cf. proof of Lemma 2.1 in [7]). The assertion then follows from the discussion preceeding the Theorem.

Let us conclude with some applications of the theorem.

1. The homotopy groups of the complex projective spaces are given by $\pi_{2}\left(\mathbb{C} P^{n}\right) \cong \mathbb{Z}$ and $\pi_{i}\left(\mathbb{C} P^{n}\right) \cong \pi_{i}\left(S^{2 n+1}\right)$ when $i>2$. The dimension of $\mathbb{C} P^{n}$ is $2 n$ and the first non-trivial Hurewicz homomorphism is bijective and so, by Theorem 3, $\mathrm{Aut}_{*}\left(\mathbb{C} P^{n}\right)=\{1\}$.

2. A Moore space $M(G, n)$ has a cellular decomposition with cells only in dimension $n$ if $G$ is a free group, and in dimensions $n$ and $n+1$ otherwise. In those dimensions the homotopy groups are $\pi_{n}(M(G, n)) \cong G$ and $\pi_{n+1}(M(G, n)) \cong G \otimes \mathbb{Z}_{2}$ (we are assuming $n>2$ ). Consequently, when $G$ is a free group or when $G \otimes \mathbb{Z}_{2}=0$ the group $\operatorname{Aut}_{*}(M(G, n))$ is trivial.

3 . Let us consider spaces $S^{n} \vee S^{n+4}$ when $n \geq 6$. The usual cellular decomposition has cells in dimensions $n$ and $n+4$ and, by the Hilton-Milnor theorem,

$$
\pi_{n}\left(S^{n} \vee S^{n+4}\right) \cong \pi_{n+4}\left(S^{n} \vee S^{n+4}\right) \cong \mathbb{Z},
$$

induced by the inclusions of $\pi_{n}\left(S^{n}\right)$ and $\pi_{n+4}\left(S^{n+4}\right)$ respectively. For that reason the Hurewicz homomorphisms in dimensions $n$ and $n+4$ are bijective; hence Aut $_{*}\left(S^{n} \vee S^{n+4}\right)$ is trivial when $n \geq 6$. By the same argument $\operatorname{Aut}_{*}\left(S^{n} \vee S^{n+5}\right)$ is 
trivial when $n \geq 7$. Note that these cases are exceptional since $A_{u t}$ of a wedge of spheres is generally non-trivial.

4. By the results of [2] the group $\operatorname{Aut}_{*}(X)$ is nilpotent and so it can be localized with respect to a set of primes. Maruyama [5] showed that Aut $_{*}$ commutes with the localization when $X$ is a simply connected finite CW-complex; i.e. the obvious map

$$
\operatorname{Aut}_{*}(X) \rightarrow \operatorname{Aut}_{*}\left(X_{(P)}\right)
$$

is a $P$-localization for any set of primes $P$. As the localization of a finite simply connected complex is countable and finite-dimensional, Theorem 3 can be applied on primary components.

\section{REFERENCES}

1. M. Arkowitz, The group of self-homotopy equivalences - a survey, in R. A. Piccinini (ed.) Group of self-equivalences and related topics, Lecture Notes in Mathematics No. 1425 (Springer-Verlag), 170-203.

2. E. Dror and A. Zabrodsky, Unipotency and nilpotency in homotopy equivalences, Topology 18 (1979), 187-197.

3. T. Ganea, A generalization of the homology and homotopy suspension, Comment. Math. Helvetici 39 (1965), 295-321.

4. P. Hilton, Homotopy theory and duality (Gordon and Breach, New York, 1965).

5. K. Maruyama, Localization of self-homotopy equivalences inducing the identity on homology, Math. Proc. Camb. Phil. Soc. 108 (1990), 291-297.

6. S. Oka, N. Sawashita and M. Sugawara, On the group of self-equivalences of a mapping cone, Hiroshima Math. J. 4 (1974), 9-28.

7. J. W. Rutter, On skeleton preserving homotopy self-equivalences of CW complexes, in R. A. Piccinini (ed.) Group of self-equivalences and related topics, Lecture notes in Mathematics No. 1425 (Springer-Verlag, 1997) 147-156.

8. J. W. Rutter, Spaces of homotopy self-equivalences, Lecture Notes in Mathematics No. 1662 (Springer-Verlag, 1997).

9. W. Shih, On the group $\epsilon[X]$ of homotopy equivalence maps, Bull. Amer. Math. Soc. 70 (1964), 361-365.

10. M. Tsukiyama, Note on self-maps inducing the identity automorphisms of homotopy groups, Hiroshima Math. J. 5 (1975), 215-222. 